\title{
Transcranial magnetic stimulation application fields
}

\author{
Ensar Demir ${ }^{1}$ (D), Oytun Erbaş²,3 \\ ${ }^{1}$ Istanbul Aydin University, Faculty of Medicine, İstanbul, Turkey \\ ${ }^{2}$ Institute of Experimental Medicine, Gebze-Kocaeli, Turkey \\ ${ }^{3}$ Department of Physiology, Medical Faculty of Demiroğlu Bilim University, Istanbul, Turkey
}

\begin{abstract}
Transcranial magnetic stimulation (TMS), which is a non-invasive procedure, has become more and more prominent with promising studies in the last two decades. With a very low rate of side effects, TMS has the potential to be effective in many diseases, particularly in psychiatric disorders such as depression, obsessive-compulsive disorder, and epilepsy. In this review, we analyze and compile studies on the development and current status of TMS and the areas where it is used or can be used. The data were obtained based on the review of about 200 studies in the literature search between January 1995 and September 2020 by scanning two online database publishing in English and one online database publishing in Turkish and English. Tens of studies examined have yielded very successful results for TMS. In the following years, it seems to enter our lives among the combined treatment methods even at worst. Undoubtedly, this cannot be ignored, but it has not been standardized yet, including two or three United States Food and Drug Administration-approved applications. Still different methodologies and protocols are available. Above all, the fact that there are unsuccessful studies or a large number of studies showing that the effect of TMS decreases significantly in the long-term indicates that it should be approached with caution.

Keywords: Epilepsy, major depressive disorder, neurodegenerative disorder, obsessive compulsive disorder, substance abuse, transcranial magnetic stimulation.
\end{abstract}

Studies on the stimulation of the human brain began with the development of electroconvulsive therapy (ECT) in the 1930s. When ECT, which was widely used in the treatment of many psychiatric diseases until the 1970s, received a serious reaction due to its adverse effects and method, different treatment methods began to be investigated. ${ }^{[1]}$ In 1985, Barker AT ${ }^{[2]}$ developed the first functional transcranial magnetic stimulation (TMS) device; however, TMS was used only in diagnosis and research for a while. It was used as a treatment method in the following years. It can affect the activity of the brain without the need for surgery or any invasive procedure and its adverse effects are much less common have made ECT an important position. ${ }^{[2,3]}$ It does not affect the memory, does not require general anesthesia and hospitalization, does not cause loss of consciousness and can be used in pregnant individuals are important factors for being an alternative to ECT. Transcranial magnetic stimulation is an imaging and treatment method based on the rule that variable magnetic field causes electric field formation, that is, Faraday's law. ${ }^{[3,4]}$ With the approval of TMS devices by the FDA (Food and Drug Administration) in 2008, TMS treatment has become widespread since 2010. ${ }^{[1]}$

According to the detailed review conducted by Burke et al. ${ }^{[5]}$ on TMS treatment in 2019, it can be used as a primary method in many areas with the increase of studies on TMS, as well as questions that need to be answered. Being a favorable neuroimaging and research technique with analysis devices such as

Correspondence: Ensar Demir. İstanbul Aydın Üniversitesi Tıp Fakültesi Öğrencisi, 34295 Sefaköy, Küçükçekmece, İstanbul, Türkiye.

e-mail: demiir.ensar@gmail.com 
electroencephalogram (EEG) and magnetic resonance imaging (MRI) and a non-invasive deep brain stimulation technique in memory research in the clinic, promising results were obtained in various fields (Alzheimer's disease, epilepsy, drug-resistant depression, preoperative imaging, diagnostic biomarkers, stroke, schizophrenia, obsessive-compulsive disorder (OCD), chronic pain, anorexia nervosa, etc.).

However, in a literature search on the database for this method, which can be considered new, although there were approximately 5,000 articles in the last five years, only 400 articles were published in the last decade, particularly in the search for adverse effects. As a result of the content scanning, much less studies have been performed on long-term adverse effects. There is a need to outline the mechanism of action at the cellular level, which is not clear yet, and studies involving long-term adverse effects, although it seems that adverse effects are rare in the short-term. ${ }^{[6]}$

\section{TRANSCRANIAL MAGNETIC STIMULATION THERAPY IN DISEASES}

According to the current guidelines published in March 2020, it is still not preferred as a first-line treatment and is particularly indicated for many drug-resistant psychiatric diseases. In addition, it is preferred in patients who develop tolerance to certain drugs and patients who require non-convulsive treatment indication. Since the first guideline published by The American Psychological Association (APA) in 2010, there has been no significant change in TMS indications. ${ }^{[7]}$

Pereira et al. ${ }^{[8]}$ examined the adverse effects of TMS treatment in 46 studies published during the period of 1990 to 2015 and observed that the risk of seizures among 410 patients was 2.9\% (95\% CI: 1.3-4.5). Therefore, the risk of adverse effects in TMS treatment is low and similar to the risk observed in healthy individuals.

\section{TRANSCRANIAL MAGNETIC STIMULATION TREATMENT IN EPILEPSY}

Epilepsy with increased cortical excitability is the most common chronic neural disease in the world. ${ }^{[3]}$ According to a study of Fiest et al. ${ }^{[9]}$ in 2017, the incidence of epilepsy worldwide is 61.4 per 100,000 per year; its prevalence is 7.60 in a population of 1,000 . In an article published by Chen et al. ${ }^{[10]}$ in 2016, the effect of TMS on the frequency of epileptic seizures and improvement in quality of life (QoL) was examined in the literature review. No change in QoL was measured in any of the seven studies. Four had a significant decrease in epileptic discharges. Only one of the total 230 participants showed an increase in the use of epileptic drugs. However, the provability of these seven studies is low.

According to another article published in 2016, TMS exerted short-term treatment effects in many studies conducted to date. ${ }^{[3]}$ In an experimental study published in 2008, a 39\% reduction in attacks was observed for four weeks after TMS treatment in nine patients with uncontrolled focal epilepsy. However, within six to eight weeks after the attacks, it returns to the pre-treatment frequency. ${ }^{[11]}$

In the review article published by VanHaerents et al. ${ }^{[12]}$ in March 2020, the results of the experiments conducted with 210 participants in total in six studies were reviewed. A study of Fregni et al. ${ }^{[13]}$ with 21 individuals (12 real, 9 placebo-controlled) showed that TMS treatment decreased the number of seizures by 50 to $70 \%$ for eight weeks and epileptiform discharges significantly over four weeks. As a result of the study conducted by Joo et al. ${ }^{[14]}$ with 35 patients, no significant decrease was observed in the frequency of seizures, and a 54.9\% decrease was observed in epileptiform discharges after TMS treatment. In a placebo-controlled study conducted by Cantello et al. ${ }^{[15]}$ with 43 patients in 2007, no significant difference was found in seizure frequency between real and placebo TMS treatment. However, a significant reduction in epileptiform discharges was observed in patients receiving real TMS treatment. Wang et al. ${ }^{[16]}$ observed that TMS treatment administered together with carbamazepine in 30 patients did not reveal a significant difference in terms of seizure frequency, compared to only carbamazepine treatment. However, as in previous studies, a significant decrease was observed in epileptiform discharges in the group receiving TMS treatment. 
However, the two recent studies examined in the article published by Sun et al. ${ }^{[17]}$ yielded more remarkable results. The first study conducted with 60 patients in 2012 examined low-density TMS and high-density TMS applications. According to the results of this study, while a decrease in seizure frequency was observed by approximately $80 \%$ in the group receiving high-intensity TMS treatment, no significant decrease was observed in the group receiving low-intensity TMS treatment. A more significant decrease was observed in the frequency of epileptiform discharges in high intensity treatment compared to low intensity treatment. The final study reviewed was conducted by Seynaeve et al..$^{[18]}$ by placebo-controlled in 11 patients. However, in this study, there was no significant decrease in seizures or epileptiform discharges compared to placebo-controlled individuals. According to another review published in 2020, if TMS was applied with certain methods, it seemed to be beneficial in reducing the frequency or severity of seizures in patients with focal epilepsy. ${ }^{[19]}$

These inconsistencies between studies can be explained by the patient-specific variables and differences in TMS methodology (treatment duration, applied magnetic frequency, intensity, focused cranial area, protocols, etc.). Therefore, to fully understand the role of TMS treatment in epilepsy treatment, further multi-center, largescale, placebo-controlled, and well-designed studies using a common methodology are needed.

\section{TRANSCRANIAL MAGNETIC STIMULATION TREATMENT IN PREGNANT INDIVIDUALS WITH EPILEPSY}

In a study of Damar et al. ${ }^{[20]}$ in 2020, TMS was applied to a 32-year-old pregnant individual for the treatment of epileptic seizures during pregnancy, temporarily reduced seizures. However, only two studies have been found in the literature on the effects of TMS treatment taken during pregnancy. One of these is the study by Eryilmaz et al., ${ }^{[21]}$ in which the development of children aged between 16 to 64 months of mothers receiving TMS treatment for depression was examined. While mothers reported that their children had delayed language development, no significant difference was found between the children of mothers who did not receive TMS treatment for depression during pregnancy and their children regarding the delay in development. The other study is one of the important studies in the literature examining the effects of TMS treatment on depression.

\section{TRANSCRANIAL MAGNETIC STIMULATION TREATMENT IN DEPRESSIVE DISORDER}

According to the World Health Organization (WHO), depression is a disease that affects more than 264 million individuals and seriously decreases the QoL. ${ }^{[22]}$ One out of every five to six individuals suffer from depressive disorder at least once in their lifetime. ${ }^{[23]}$ Depression ranks third in the WHO's world disease burden list in 2008, and it is predicted to rise to the first place in 2030. ${ }^{[24]}$ There are effective treatments for these and similar mental disorders; however, almost $80 \%$ of individuals in low- and middle-income countries do not receive any treatment. ${ }^{[25]}$

Pascual-Leone et al. ${ }^{[26]}$ in 1996, active rapid rTMS (repetitive TMS) was applied to 17 patients to the left and right dorsolateral prefrontal cortex (DLPFC), placebo rTMS in the same areas, and active TMS to the vertex region in the same areas for five months. The most positive response was obtained in the active fast rTMS application applied to the left DLPFC, significantly better than other methods. Eleven of the 17 patients showed improvement with this application; however, these improvements decreased within two weeks.

Grunhaus et al. ${ }^{[27]}$ in the study published in 2000, 40 patients with major depressive disorder (MDD) who were referred to ECT were randomly referred to ECT and rTMS. As a result of this 20-day study, ECT was a stronger treatment in patients with MDD in general. However, both treatment methods yielded similar results in patients with MDD without psychosis.

Sayar et al. ${ }^{[28]}$ in 2013 applied TMS treatment to the left DLPFC in combination with antidepressant treatment in 65 patients with treatment-resistant depression with a mean age of 66.6 years. After the treatment, more than $50 \%$ improvement was observed in 38 patients according to the Hamilton Rating Scale for Depression (HRSD), and remission was observed 
in 19 of these 38 patients. Partial improvement was observed in all of the remaining patients according to the HRSD. No adverse effects or worsening were reported in this study.

A review comparing the effect of rTMS on drug-resistant depression with ECT and placebo was published in 2016. This review examined randomized-controlled trials (RCTs) published from 1994 to 2015. Twenty-three RCTs compared rTMS with placebo, six RCTs compared rTMS with ECT. There was a $10 \%$ absolute difference in both response and remission between rTMS and placebo. However, rTMS studies against ECT yielded statistically significant results in favor of ECT. As shown in many different studies, rTMS has a short-term effect as a result of followup studies. Evidence quality were moderate to low in when rTMS-placebo comparative studies, while rTMS-ECT is at an intermediate level in comparative studies. ${ }^{[29]}$

\section{TRANSCRANIAL MAGNETIC STIMULATION TREATMENT AND EFFECTS IN PREGNANCY DEPRESSION}

Sayar et al. ${ }^{[30]}$ in 2013, they applied high frequency rTMS to the left PFC in 29 pregnant individuals with drug-resistant depression, six days a week for 18 days in total. Hamilton Rating Scale for Depression was used to evaluate the results, and the depression levels of the patients from the last rTMS session were evaluated one week after the last rTMS session. The mean age of the patients was $32.7 \pm 3.7$ years (range, 25 to 39), and the mean week of gestation was $14.3 \pm 8.3$ weeks (range, 5 to 32 ). At the end of the treatment, in 12 patients (41.1\%), the HRDS scale decreased by more than $50 \%$ and improved mood. Remission was observed in six patients (20.7\%), partial response in 10 patients (34.5\%), and worsening on the HRDS scale in one patient (3.4\%). No adverse effects were reported at the end of the study. A total of 23 of the 29 pregnant individuals delivered 25 healthy newborns. Newborns did not show any abnormalities either. Physical and neurological examination results revealed that pregnancy and newborn screening test results and hearing evaluation were normal. No abnormality was found in all newborns during the mean follow-up period of $24.8 \pm 18.5$ months. Six fetuses are still under intensive follow-up and no signs of intrauterine growth retardation were observed.

In additional study published in 2018, TMS treatment was applied for major depressive disorder in 22 pregnant individuals, 11 of whom were active and 11 of whom were in the placebo group. A significant reduction in the HRDS scale was observed in the active group compared to the placebo group. The response rate of the active group was $81.82 \%$, while the response rate of the placebo group was $45.45 \%$. Remission rates were $18.18 \%$ for the placebo group and $27.27 \%$ for the active group. Similar growth was observed in all fetuses in 18 individuals throughout the study. In addition, three preterm births (PTBs) were seen in the active group; however, a larger study is needed to show that this difference is statistically significant. In the literature, there was only one case who received high-frequency TMS after 31 weeks of gestation and delivered at the age of 36 years. One newborn in the active group had a brachial plexus injury at the initial pediatric evaluation; however, it was determined that this was a result of shoulder dystocia, which was not related to TMS. ${ }^{[31]}$

\section{TRANSCRANIAL MAGNETIC STIMULATION IN OBSESSIVE COMPULSIVE DISORDER}

Obsessive-compulsive disorder is one of the frequently encountered serious and chronic neurological diseases. The treatment of OCD, which can seriously reduce the QoL of the patient if it is severe, is generally continued with pharmacological agents. Unfortunately, most patients are resistant to drug therapy. Therefore, different and new treatment methods are needed. It affects approximately $2.3 \%$ of individuals at some point in their lives and half of the diagnosed patients show symptoms before the age of 20 . It is unusual for symptoms to begin after the age of 35 years. ${ }^{\text {[2-34] }}$

In 2001, Alonso et al. ${ }^{[35]}$ applied rTMS to right PFC in a double-blind and placebo-controlled study. While 10 patients were the real rTMS group, eight patients were designated as the placebo group. As a result of applying low frequency and 110\% motor threshold sensitivity 
during 18 sessions of $20 \mathrm{~min}$, no significant difference was observed between the participants. However, it is thought that independent factors such as the age difference between the groups, the small sample size and the brain region where the procedure is applied are effective in obtaining such a resulk.

In 2006, rTMS treatment was applied to the supplementary motor cortex (SMC) on 10 patients. Five patients have OCD, three patients have Tourette syndrome (TS) and two patients have OCD-comorbid TS. After the application, an improvement was observed from the first week. This process continues in a stable manner during the three-month follow-up period; however, considering that it is not placebocontrolled and the sample size is relatively low, further large-scale RCTs are needed. ${ }^{[36]}$

In March 2010, Mantovani et al. ${ }^{[37]}$ conducted a detailed study on 21 drug-resistant patients. The application was planned in two stages and the first stage is a four-week double-blind study, while Stage 2 is a four-week open-label study. Patients were randomly divided into placebo and active groups. Treatment was applied five days a week for four weeks, targeting the pre-SMC (supplementary motor cortex) area. Eighteen of the patients completed the first stage. Three patients withdrew from the study prior to study. Those included in the active group were assumed to be randomly classified as non-responders and those in the placebo group as non-responders, based on the worst-case scenario analysis who withdrew from the study. The response rate at Week 4 was $54 \%(6 / 11)$ in the active group; it was $20 \%$ in the placebo group $(2 / 10)$. With 18 complements, the proportions were $67 \%(6 / 9)$ in the active group and $22 \%(2 / 9)$ in the placebo group. According to the Yale-Brown ObsessiveCompulsive Scale (YBOCS), the active group showed a $25 \%$ reduction in OCD symptoms in the first four weeks, and the placebo group showed a $12 \%$ reduction. In Phase 2 , which was planned as an open-label study, rTMS treatment was recommended to all patients for four weeks. Twelve patients agreed to receive active rTMS for four weeks. Initially, four patients with active rTMS group (to be called continuously active) showed greater improvement in YBOCS (from $17.7 \pm 2.6$ to $14.5 \pm 3.6$ ) by Week 8 . However, there was no significant change in OCD symptoms of eight individuals who were originally in the placebo group after rTMS taken between Weeks 4 and 8 (YBOCS slightly increased from $25.6 \pm 7$ to $26.3 \pm 8.5$ ). In the analyzes performed on these two groups, the correlation between improvement of OCD symptoms and clinical improvement of the continuously active group is significant. In the placebo group, there was no significant change in OCD symptoms and depression, with a significant improvement in general anxiety (according to the HAM-A [Hamilton Anxiety Rating Scale]). Eight patients who responded during the three-month follow-up period (four active rTMS for 8 weeks; two active rTMS for four weeks; one receiving placebo for four weeks; the other receiving four weeks placebo + four weeks active rTMS) continued to meet the response criteria. Six patients who responded to active rTMS showed a 51\% decrease compared to the baseline YBOCS.

This study is the first study on drug-resistant OCD and although it has yielded positive results in treatment, it requires more studies in this direction. The main limitations of the results of this study are the small size of the study sample, the difference between the sham rTMS used in the placebo group and the fact that the majority of the patients had comorbid depression), and less frequently SMC and orbitofrontal cortex (OFC). In a review published in 2010, one of the British publication databases was reviewed from 1966 to 2010 and TMS treatment in OCD was examined. As a result of a study of Jaafari et al., ${ }^{[38]} \mathrm{SMC}$ and OFC are the most promising areas for OCD treatment; however, larger studies are still needed. Another review published in 2019 indicated that SMC and OFC should be the target area in OCD treatment as a result of six database scans. ${ }^{[39]}$

While evaluating all the studies on the therapeutic efficacy of TMS including OCD, the non-standardization of study conditions (frequency, number of stimulations, duration, session amount, device used, targeted area, etc.) and clinical heterogeneity (age of onset of OCD, current treatment status, patient age, the severity of symptoms, comorbid diseases, etc.) need attention. Although many conditions mentioned above determine the response to TMS treatment, the mild or moderate severity of the disease significantly affects it. ${ }^{[4]}$ 
In recent years, several studies have been published showing that the application of deep TMS (dTMS) in the treatment of OCD can yield effective results in different regions. Deep TMS is an additional treatment method rather than being an alternative to rTMS in that it can affect subcortical brain regions and internal neuronal regions such as anterior cingulate cortex (ACC). However, due to the small number of studies focused on dTMS, dTMS remains unclear for now. ${ }^{[39-41]}$

One of the few studies focusing on dTMS that has minimized the reasons for inconsistency mentioned above was published in 2019. This study was planned in 11 centers and randomly enrolled 99 OCD patients into the high-frequency dTMS or placebo group, five days a week for six weeks, focusing on the ACC and medial prefrontal cortex (mPFC). The follow-up phase lasted four weeks and was evaluated using the YBOCS criteria. Five individuals withdrew from the study prior to study initiation. There were 35 patients each in both active and placebo groups reporting adverse effects. Adverse effects manifested as the most frequently reported headache in the literature (active $37.5 \%$ vs. placebo $35.3 \%$ ), and other symptoms are typical effects seen in previous TMS studies. Only one patient reported serious adverse effects as suicidal thoughts. However, the patient stated that he had these thoughts before the study started, and it was decided that hospitalization would be appropriate. The decrease in YBOCS values (active dTMS: 6 points; placebo: 3.3 points) in patients receiving active dTMS treatment was significantly higher than in the placebo group. Response rates were $38.1 \%$ and $11.1 \%$, respectively. At one month of follow-up, these rates were $45.2 \%$ and $17.8 \%$, respectively. This $\mathrm{RCT}$ is the first study in which multi-center dTMS application on mPFC and ACC was performed. ${ }^{[42]}$

Additional non-multicenter study on ACC and mPFC with a smaller sample; however, with the same results, was also conducted by Carmi et al. ${ }^{[43]}$ published in 2018. In this study, high frequency dTMS was applied to a patient group of 16 individuals and showed significant differences compared to the placebo group of 14 individuals. Although these results are promising, similar results need to be confirmed over and over.
Most of the studies on TMS have focused on three diseases (epilepsy/depression/OCD) examined so far, however, as stated before, there is no standardization in terms of either methodology or protocols for TMS yet. Moreover, the mechanism of action of TMS at the cellular level is unclear. The FDA approved rTMS and dTMS only in the treatment of major depressive disorder (MDD) and OCD in 2008 and 2018, respectively. Most of the studies we examine for OCD and depression have fundamental differences, even in a narrow perspective (Table 1). Although dTMS treatment has been an option in the treatment of opioid or drug addiction in the last few years, it is not at a sufficient point for approval. However, studies and results on topics that will be examined hereafter, including opioid addiction, are not yet at the required level. ${ }^{[43]}$

\section{TRANSCRANIAL MAGNETIC STIMULATION IN NEURODEGENERATIVE DISEASES}

\section{Transcranial magnetic stimulation in Alzheimer's Disease}

Alzheimer's disease (AD) is a disease that occurs with the accumulation of amyloid plaques in the brain and manifested as paranoia, memory loss and confusion. According to WHO data, AD, which affects approximately 50 million individuals in the world, was the $14^{\text {th }}$ most common cause of death in 2000, and increased to fifth place in 2016 as the cause of death of 2 million individuals. ${ }^{[44]}$ According to the Turkish Neurology Association, more than 300,000 in Turkey and according to $\mathrm{NIH}$ (National Institutes of Health) data, it is thought that the number of individuals suffering from this disease, which affects more than 5.5 million individuals in the United States of America (USA), would double in 2050. ${ }^{[45,46]}$

In recent years, studies on the prognosis or treatment of $\mathrm{AD}$ have yielded unsuccessful results, and new methods such as TMS have started to be used. Although existing pharmacological treatments do not give positive results in most patients, they are used due to the lack of alternative therapies. ${ }^{[47,48]}$ In 2010 , Cotelli et al. ${ }^{[49]}$ randomly divided 10 patients with Alzheimer's into two groups and determined one as rTMS and the other as placebo group. For the placebo group, the fake rTMS is in the first two weeks, then the 


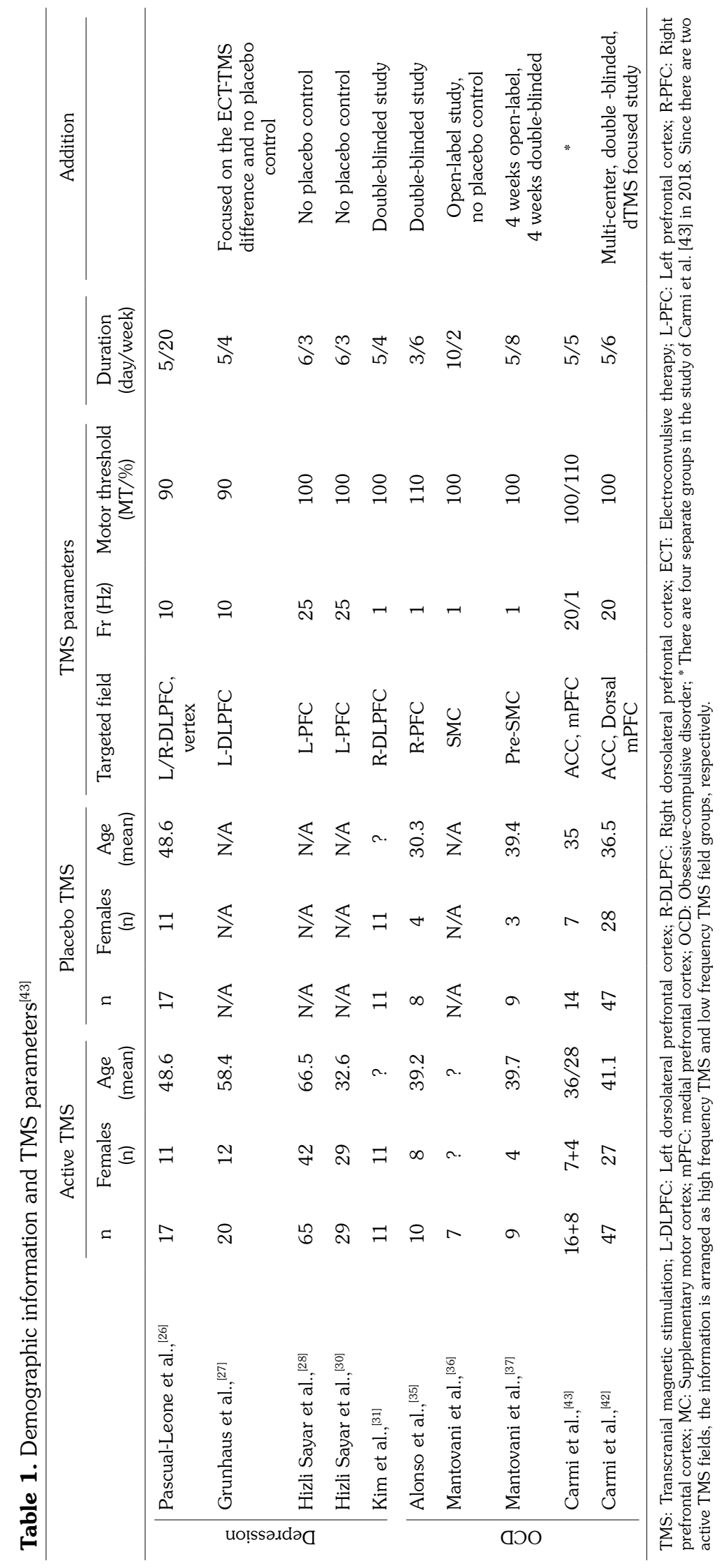


real rTMS is the following two weeks applied and real rTMS to the left DLPFC for five days a week for four weeks applied only to the other group. At the end of the study, a significant increase was observed only in the percentages of auditory sentences consciousness. This improvement continued after eight weeks of follow-up. Two weeks of rTMS treatment yielded the same results as four weeks of rTMS treatment. A prospective, placebo-controlled, double-blind RCT was studied with 30 patients in 2017. Significant improvement was observed in the patients who evaluated according to the ADAS-cog (Alzheimer's Disease Assessment Scale-Cognitive Subscale) score at the $6^{\text {th }}$ and $12^{\text {th }}$ weeks after the study. However, the significant improvement of patients in the mild stage of the disease compared to those in the middle stage is one of the striking points of this study. As in the early stage in $\mathrm{AD}$, it increases our hopes for TMS both in terms of diagnosis and treatment. ${ }^{[48-50]}$

Transcranial magnetic stimulation gives as much hope in the diagnostic field, not just treatment in AD. ${ }^{[49-51]}$ In a study in 2018, 120 patients were subjected to a three-stage evaluation (only clinical/clinical + amyloid markers/clinical + TMS). Two blinded neurologists also reviewed each stage for diagnostic confidence. As a result of the studies, TMS has the potential to compete with amyloid markers. ${ }^{[52]}$ In March 2019, in a review of Lin et al., ${ }^{[53]}$ the results of rTMS treatment received by 231 patients from 12 studies selected as a result of literature review were examined. Significant improvements in cognitive abilities were achieved as a result of long-term treatments in different regions. Although the use of TMS as an alternative or combined treatment method in Alzheimer's disease has yielded positive results in a few studies, it is still approached with caution.

\section{Transcranial magnetic stimulation in Parkinson's disease (PD)}

Parkinson's disease, which shows pathological progression with the loss of dopaminergic pathways in Lewy bodies and substantia nigra and is classified as a movement disorder, affects one to two individuals out of every 1,000 individuals. A total of $1 \%$ of the population over the age of 60 suffers from this disease. As the disease progresses, neocortical and cortical regions are also affected by Lewy bodies. Thus, PD manifests itself with both motor and non-motor symptoms. Although environmental factors and genetics (5 to $10 \%$ ) are play a role in etiology, PD is still a complex and heterogeneous neurodegenerative disease. ${ }^{[54-56]}$

In a study conducted in January 2018, 19 patients (mean age 69.1 \pm 8.4 years; age range, 52 to 84 years) were divided into placebo and real rTMS groups. No serious and long-term adverse effects were reported during this single-blinded study where high frequency rTMS was applied to motor cortex, SMC and DLPFC. Motor symptoms improved significantly as a result of applications to SMC and M1 (primary motor cortex), and no significant improvement was observed in mood disorders. ${ }^{[57]}$

Similar results were obtained in a placebocontrolled multi-center double-blind RCT published in 2016. In the study, which started with 61 individuals, all stages of 50 patients were completed. M1, DLPFC and placebo were administered alone or in combination with various patient groups. The patients were evaluated with the Unified Parkinson's Disease Rating ScaleIII (UPDRS-III) and their mood symptoms with HRSD at the beginning, at the first week and at one, three, and six months of treatment. The rTMS treatment applied to the M1 region provided a significant improvement in motor function compared to the placebo group. However, no improvement in mood or motor functions was observed in the M1 and DLPFC combination or in the DLPFC group alone. In this study, simultaneous stimulation was given to different areas at the same time in some combination groups and there was no significant improvement. Contrary to what is expected, simultaneous stimulation may not have a combined total effect and block each other's effect; however, there is not enough information about the neurophysiology of simultaneous stimulation. ${ }^{[58]}$

In another study by Cohen et al. ${ }^{[59]}$ published in 2018, the results are in contrast to the above studies. Twenty-one patients were designated as deep rTMS (rDTMS) and 21 patients as placebo. The group receiving deep rTMS showed improvement in both depression symptoms (Becks depression inventory-BDI) and motor function 
(UPDRS); however, this improvement was not significant compared to the placebo group. Similar results were also seen in another study published in 2019 by Trung et al. ${ }^{[60]}$

Examination of several review studies published on the effect of TMS on PD in the literature, rTMS applied at a frequency suitable for certain target regions and in sessions exhibits positive and significant differences at least in motor functions. ${ }^{[61,62]}$ Indeed, three pivotal multicenter, double-blind, placebo-controlled RCTs conducted in Japan achieved almost common results in this regard, and this again increases our hopes for rTMS in the treatment of PD. As previously stated, more large-scale studies are required to accept rTMS as a treatment method. ${ }^{[63]}$

\section{TRANSCRANIAL MAGNETIC STIMULATION IN HUNTINGTON'S DISEASE AND ALS (AMYOTROPHIC LATERAL SCLEROSIS)}

Very few rTMS studies conducted in movement disorders other than PD have not been successful in literature. In the last two decades, few studies on non-PD movement disorders and TMS have been published. In these studies, TMS was tested as an imaging method rather than a treatment method. ${ }^{[64]}$

\section{TRANSCRANIAL MAGNETIC STIMULATION IN SCHIZOPHRENIA, ANXIETY, BIPOLAR DISORDER (BD), AND SUBSTANCE ADDICTION}

\section{Schizophrenia}

Schizophrenia is a complex, heterogeneous cognitive syndrome that might be caused by genetic or environmental factors and affected 21 million individuals in 2016. A little different from other psychiatric diseases, it is not only a dysfunction in certain brain regions, also a common disorder in the brain. ${ }^{[65,66]}$

In a review examining $41 \mathrm{RCT}$ s in the literature, TMS applied to the temporoparietal region significantly improved positive symptoms compared to placebo. However, the quality of evidence of most of the studies reviewed has been evaluated as very low and, therefore, nothing about the clinical use of TMS cannot be said for this study. ${ }^{[67]}$ In another review published in 2019, in 12 of 30 studies examined, TMS was found to have a significant effect on the improvement of positive symptoms, while 18 studies did not find sufficient evidence.

As in the previous review, the quality of the evidence was evaluated as very low in most of the studies reviewed here. ${ }^{[68]}$ In the studies reviewed in these reviews, improvement in positive symptoms was often seen only in auditory hallucinations. Other positive symptoms need further study. These small inconsistencies in the study results are further exacerbated by studies showing that TMS can only affect negative symptoms (again with minor discrepancies). ${ }^{69,70]}$ In addition, the existence of multi-center, placebo-controlled RCTs indicating that the application of high-frequency rTMS to the left DLPFC does not show a significant difference indicates that TMS is too early to be used for treatment in schizophrenia. ${ }^{[71]}$

\section{Anxiety}

Anxiety usually occurs as a comorbid to other psychiatric diseases, as considered and examined by Mantovani et al. ${ }^{[36]}$ in OCD. Most of the limited studies related to TMS focus not directly on anxiety, also on diseases in which anxiety is comorbid. In a review examined by Kozel ${ }^{[72]}$ in 2018, rTMS treatment provided significant improvements in anxiety and this improvement continued during the three-month follow-up period. Other studies that observe similar results are available in the literature; however, the high heterogeneity among these few studies necessitates multi-center studies with a single protocol. ${ }^{[3,74]}$

\section{Bipolar disorder}

Bipolar disorder is a psychiatric disease that affects $2.5 \%$ of the world population and the suicide rates are 20 to 30 times higher than the normal population. ${ }^{[72,75]}$ The therapeutic effect of TMS in BD has been evaluated over symptoms in very few studies. Although there are more results showing that it improves the depression seen in $\mathrm{BD}$, the opposite results are also remarkable. Transcranial magnetic stimulation studies on mania give much more complex and inconsistent results. ${ }^{[72,75,76]}$ Although many treatment methods have been subjected to experiments and studied, the only treatment method that can reduce suicide 
rates in $\mathrm{BD}$ is Lithium therapy for now. ${ }^{[75]}$ As we mentioned in the previous titles, additional studies are needed in $\mathrm{BD}$.

\section{Substance abuse}

The use of addictive substances is common worldwide. In the USA alone, more than 21.9 million individuals over the age of 12 are known to be addicted to illegal drugs. ${ }^{[77]}$ Ecstasy (MDMA (3-methoxy-4,5-methylenedioxyamphetamine; 5 -methoxy-MDA)) is used by 1 to $4 \%$ of the adult population in most European countries, although it is the second most widely used drug in Europe. However, the level of addiction in the UK (United Kingdom) is much more dramatic compared to other European countries. Cocaine use increased five times between 1994 and $2004{ }^{\text {[78] }}$ Addiction treatment application rates have increased six times from 1999 to 2009, and the mortality rate due to opioid overdose has increased from 3.7 per 10,000 in 2011 to 7.3 by $94 \%$ in $2015 .{ }^{.79]}$

Current pharmacological treatments have a limited effect in reducing addiction and abstinence and a high relapse rate. Despite significant progress in understanding the neurological fundamentals of addiction, therapeutic options are limited. This is why TMS has a potential treatment method. ${ }^{[74]} \mathrm{A}$ 2017 study investigating the effect of TMS on substance seeking behavior examined 199 patients. Active TMS treatment focused on the right DLPFC gave more successful results compared to placebo. ${ }^{[80]}$ There are other studies that have yielded results in contrast to this study, which is a small-scale study with very low evidence. Similar to the 2018 study of Azevedo and Mammis, ${ }^{[11]}$ who were reported to be unable to improve the recurrence rates of TMS. Different brain regions may need to be targeted according to the substance to which the addicted substance, for example, targeting the $\mathrm{PFC}$ region for cocaine addiction or the DLPFC region for tobacco addiction has been shown to yield positive results. ${ }^{[80,82]}$ The most important problem in addiction treatment is high relapse rates. There are also studies showing that these rates can be reduced with TMS treatment; however, confirmatory and detailed studies are required to make definitive conclusions. ${ }^{[81]}$

\section{ADDITIONAL APPLICATION FIELDS}

Transcranial magnetic stimulation has come into prominence in recent years as a potential treatment method in a wide variety of fields and has been the subject of studies. Despite the heterogeneity and different protocols in studies on neuropathic, chronic pain or migraine, short- or long-term pain relief effects have been observed. ${ }^{[83-85]}$ There are several studies that state that it improves communication skills or cognitive functions in the treatment of autism. ${ }^{86,87]}$ Or, it is known by an increasing number of studies that significant improvements have been observed in the treatment of tinnitus. ${ }^{[8]}$ Transcranial magnetic stimulation is used not only as a treatment method, is also an imaging method in which brain functions are mapped. ${ }^{[89]}$

In conclusion, after this study, where we focused specifically on the therapeutic efficacy of TMS, we found that the general opinion tends to have therapeutic benefit. Additional reason for this trend may be the relatively low number of unsuccessful studies compared to successful ones. However, as we have stated many times, our lack of knowledge about TMS is more than thought. Still, the number and duration of sessions to be applied to each disease, frequency level, device type, TMS type (rTMS, dTMS etc.), brain region to be targeted (DLPFC, PFC, SMC etc.), which phase of the disease will be applied, protocols, experimental methodology are very broad and show diversity. In addition, questions such as its function in acute treatment, long-term results and adverse effects, and the ability to be combined in treatments also clearly show the need for multicenter, placebo-controlled RCTs with a large sample. Of course, the considerable positive and promising results achieved so far cannot be ignored; however, it is still useful to treat the standardized one with caution until a common protocol is reached..$^{[3]}$

\section{Declaration of conflicting interests}

The author declared no conflicts of interest with respect to the authorship and/or publication of this article.

\section{Funding}

The author received no financial support for the research and/or authorship of this article. 


\section{REFERENCES}

1. Horvath JC, Perez JM, Forrow L, Fregni F, PascualLeone A. Transcranial magnetic stimulation: a historical evaluation and future prognosis of therapeutically relevant ethical concerns. J Med Ethics 2011;37:137-43.

2. Barker AT, Jalinous R, Freeston IL. Non-invasive magnetic stimulation of human motor cortex. Lancet 1985;1:1106-7.

3. Noohi S, Amirsalari S. History, studies and specific uses of repetitive transcranial magnetic stimulation (rTMS) in treating Epilepsy. Iran $\mathrm{J}$ Child Neurol 2016;10:1-8.

4. Kingman R, Rowland SC, Popescu S. An experimental observation of Faraday's law of induction. American Journal of Physics 2002;70:595-8.

5. Burke MJ, Fried PJ, Pascual-Leone A. Transcranial magnetic stimulation: Neurophysiological and clinical applications. Handb Clin Neurol 2019;163:73-92.

6. Zorzo C, Banqueri M, Higarza SG, Pernía AM, Arias JL. Current State of Transcranial Magnetic Stimulation and its use in Psychiatry. Actas Esp Psiquiatr 2019;47:110-20.

7. Transcranial Magnetic Stimulation Guideline. United Healthcare ${ }^{\circledR}$ Services Inc. Guideline Number: MPG313.05, 2020. Available at: https://www. uhcprovider.com/content/dam/provider/docs/public/ policies/medadv-guidelines/t/transcranial-magneticstimulation.pdf

8. Pereira LS, Müller VT, da Mota Gomes M, Rotenberg A, Fregni F. Safety of repetitive transcranial magnetic stimulation in patients with epilepsy: A systematic review. Epilepsy Behav 2016;57:167-76.

9. Fiest KM, Sauro KM, Wiebe S, Patten SB, Kwon $\mathrm{CS}$, Dykeman J, et al. Prevalence and incidence of epilepsy: A systematic review and meta-analysis of international studies. Neurology 2017;88:296-303.

10. Chen R, Spencer DC, Weston J, Nolan SJ. Transcranial magnetic stimulation for the treatment of epilepsy. Cochrane Database Syst Rev 2016;8:CD011025.

11. Kanai R, Chaieb L, Antal A, Walsh V, Paulus W. Frequency-dependent electrical stimulation of the visual cortex. Curr Biol 2008;18:1839-43.

12. VanHaerents S, Chang BS, Rotenberg A, PascualLeone A, Shafi MM. Noninvasive brain stimulation in Epilepsy. J Clin Neurophysiol 2020;37:118-30.

13. Fregni F, Otachi PT, Do Valle A, Boggio PS, Thut G, Rigonatti SP, et al. A randomized clinical trial of repetitive transcranial magnetic stimulation in patients with refractory epilepsy. Ann Neurol 2006;60:447-55.

14. Joo EY, Han SJ, Chung SH, Cho JW, Seo DW, Hong $\mathrm{SB}$. Antiepileptic effects of low-frequency repetitive transcranial magnetic stimulation by different stimulation durations and locations. Clin Neurophysiol 2007;118:702-8.

15. Cantello R, Rossi S, Varrasi C, Ulivelli M, Civardi $\mathrm{C}$, Bartalini $\mathrm{S}$, et al. Slow repetitive TMS for drug- resistant epilepsy: clinical and EEG findings of a placebo-controlled trial. Epilepsia 2007;48:366-74.

16. Wang $\mathrm{X}$, Yang $\mathrm{D}$, Wang S, Zhao X, Zhang $\mathrm{L}$, Chen $\mathrm{Z}$, et al. Effects of low-frequency repetitive transcranial magnetic stimulation on electroencephalogram and seizure frequency in 15 patients with temporal lobe epilepsy following dipole source localization. Neural Regeneration Research 2008;3:1257-60.

17. Sun W, Mao W, Meng X, Wang D, Qiao L, Tao $\mathrm{W}$, et al. Low-frequency repetitive transcranial magnetic stimulation for the treatment of refractory partial epilepsy: a controlled clinical study. Epilepsia 2012;53:1782-9.

18. Seynaeve L, Devroye A, Dupont P, Van Paesschen W. Randomized crossover sham-controlled clinical trial of targeted low-frequency transcranial magnetic stimulation comparing a figure- 8 and a round coil to treat refractory neocortical epilepsy. Epilepsia 2016;57:141-50.

19. Tsuboyama M, Kaye HL, Rotenberg A. Review of Transcranial Magnetic Stimulation in Epilepsy. Clin Ther 2020;42:1155-68.

20. Damar U, Lee Kaye H, Smith NA, Pennell PB, Rotenberg A. Safety and tolerability of repetitive transcranial magnetic stimulation during pregnancy: a case report and literature review. J Clin Neurophysiol 2020;37:164-9.

21. Eryılmaz G, Sayar GH, Özten E, Gül IG, Yorbik Ö, Işiten $\mathrm{N}$, et al. Follow-up study of children whose mothers were treated with transcranial magnetic stimulation during pregnancy: preliminary results. Neuromodulation 2015;18:255-60.

22. GBD 2017 Disease and Injury Incidence and Prevalence Collaborators. Global, regional, and national incidence, prevalence, and years lived with disability for 354 diseases and injuries for 195 countries and territories, 1990-2017: a systematic analysis for the Global Burden of Disease Study 2017. Lancet 2018;392:1789-858.

23. Bromet E, Andrade LH, Hwang I, Sampson NA, Alonso J, de Girolamo G, et al. Cross-national epidemiology of DSM-IV major depressive episode. BMC Med 2011;9:90.

24. WHO. The global burden of disease: 2004 update. Geneva: World Health Organization; 2008.

25. Wang PS, Aguilar-Gaxiola S, Alonso J, Angermeyer MC, Borges G, Bromet EJ, et al. Use of mental health services for anxiety, mood, and substance disorders in 17 countries in the WHO world mental health surveys. Lancet 2007;370:841-50.

26. Pascual-Leone A, Rubio B, Pallardó F, Catalá MD. Rapid-rate transcranial magnetic stimulation of left dorsolateral prefrontal cortex in drug-resistant depression. Lancet 1996;348:233-7.

27. Grunhaus L, Dannon PN, Schreiber S, Dolberg OH, Amiaz R, Ziv R, et al. Repetitive transcranial magnetic stimulation is as effective as electroconvulsive 
therapy in the treatment of nondelusional major depressive disorder: an open study. Biol Psychiatry 2000;47:314-24.

28. Hizli Sayar G, Ozten E, Tan O, Tarhan N. Transcranial magnetic stimulation for treating depression in elderly patients. Neuropsychiatr Dis Treat 2013;9:501-4.

29. Razza LB, Moffa AH, Moreno ML, Carvalho AF, Padberg F, Fregni $F$, et al. A systematic review and meta-analysis on placebo response to repetitive transcranial magnetic stimulation for depression trials. Prog Neuropsychopharmacol Biol Psychiatry 2018;81:105-13.

30. Hızlı Sayar G, Ozten E, Tufan E, Cerit C, Kağan G, Dilbaz $\mathrm{N}$, et al. Transcranial magnetic stimulation during pregnancy. Arch Womens Ment Health 2014;17:311-5.

31. Kim DR, Wang E, McGeehan B, Snell J, Ewing $G$, Iannelli $C$, et al. Randomized controlled trial of transcranial magnetic stimulation in pregnant women with major depressive disorder. Brain Stimul 2019;12:96-102.

32. Drubach DA. Obsessive-compulsive disorder. Continuum (Minneap Minn) 2015;21:783-8.

33. Goodman WK, Grice DE, Lapidus KA, Coffey BJ. Obsessive-compulsive disorder. Psychiatr Clin North Am 2014;37:257-67.

34. Diagnostic and statistical manual of mental disorders: DSM-5. 5th ed. Washington: American Psychiatric Publishing; 2013. p. 237-42.

35. Alonso P, Pujol J, Cardoner N, Benlloch L, Deus $\mathrm{J}$, Menchón $\mathrm{JM}$, et al. Right prefrontal repetitive transcranial magnetic stimulation in obsessivecompulsive disorder: a double-blind, placebocontrolled study. Am J Psychiatry 2001;158:1143-5.

36. Mantovani A, Lisanby SH, Pieraccini F, Ulivelli M, Castrogiovanni $\mathrm{P}$, Rossi S. Repetitive transcranial magnetic stimulation (rTMS) in the treatment of obsessive-compulsive disorder (OCD) and Tourette's syndrome (TS), International Journal of Neuropsychopharmacology 2006;9:95-100.

37. Mantovani A, Simpson HB, Fallon BA, Rossi S, Lisanby SH. Randomized sham-controlled trial of repetitive transcranial magnetic stimulation in treatment-resistant obsessive-compulsive disorder. Int J Neuropsychopharmacol 2010;13:217-27.

38. Jaafari N, Rachid F, Rotge JY, Polosan M, El-Hage $\mathrm{W}$, Belin D, et al. Safety and efficacy of repetitive transcranial magnetic stimulation in the treatment of obsessive-compulsive disorder: a review. World J Biol Psychiatry 2012;13:164-77.

39. Rapinesi C, Kotzalidis GD, Ferracuti S, Sani G, Girardi P, Del Casale A. Brain Stimulation in ObsessiveCompulsive Disorder (OCD): A Systematic Review. Curr Neuropharmacol 2019;17:787-807.

40. Lusicic A, Schruers KR, Pallanti S, Castle DJ. Transcranial magnetic stimulation in the treatment of obsessive-compulsive disorder: current perspectives. Neuropsychiatr Dis Treat 2018;14:1721-36.
41. Carmi, L, Al yagon U, Dar R, Zohar J, Zangen A. Deep Transcranial Magnetic Stimulation (Tms) in Obsessive Compulsive Disorder (Ocd) Patients. European Psychiatry 2015;30:1.

42. Carmi L, Tendler A, Bystritsky A, Hollander E, Blumberger DM, Daskalakis J, et al. Efficacy and safety of deep transcranial magnetic stimulation for obsessive-compulsive disorder: A prospective multicenter randomized double-blind placebocontrolled trial. Am J Psychiatry 2019;176:931-8.

43. Carmi L, Alyagon U, Barnea-Ygael N, Zohar J, Dar R, Zangen A. Clinical and electrophysiological outcomes of deep TMS over the medial prefrontal and anterior cingulate cortices in OCD patients. Brain Stimul 2018;11:158-65.

44. The top 10 causes of death. Available at: https:// www.who.int/news-room/fact-sheets/detail/the-top10-causes-of-death

45. Available at: Dementia. https://www.who.int/newsroom/fact-sheets/detail/dementia

46. Available at: Alzheimer Hastalığı. https://www. noroloji.org.tr/menu/94/alzheimer-hastaligi

47. Weiler M, Stieger KC, Long JM, Rapp PR. Transcranial magnetic stimulation in alzheimer's disease: are we ready? eNeuro 2020;7:ENEURO.0235-19.2019.

48. Rajji TK. Transcranial magnetic and electrical stimulation in Alzheimer's disease and mild cognitive impairment: A review of randomized controlled trials. Clin Pharmacol Ther 2019;106:776-80.

49. Cotelli M, Calabria M, Manenti R, Rosini S, Zanetti $\mathrm{O}$, Cappa SF, et al. Improved language performance in Alzheimer disease following brain stimulation. $J$ Neurol Neurosurg Psychiatry 2011;82:794-7.

50. Zhao J, Li Z, Cong Y, Zhang J, Tan M, Zhang H, et al. Repetitive transcranial magnetic stimulation improves cognitive function of Alzheimer's disease patients. Oncotarget 2017;8:33864-71.

51. Koch G, Martorana A, Caltagirone C. Transcranial magnetic stimulation: Emerging biomarkers and novel therapeutics in Alzheimer's disease. Neurosci Lett 2020;719:134355.

52. Benussi A, Alberici A, Ferrari C, Cantoni V, Dell'Era $\mathrm{V}$, Turrone $\mathrm{R}$, et al. The impact of transcranial magnetic stimulation on diagnostic confidence in patients with Alzheimer disease. Alzheimers Res Ther 2018;10:94.

53. Lin Y, Jiang WJ, Shan PY, Lu M, Wang T, Li RH, et al. The role of repetitive transcranial magnetic stimulation (rTMS) in the treatment of cognitive impairment in patients with Alzheimer's disease: A systematic review and meta-analysis. J Neurol Sci 2019;398:184-91.

54. Tysnes OB, Storstein A. Epidemiology of Parkinson's disease. J Neural Transm (Vienna) 2017;124:901-5.

55. Simon DK, Tanner CM, Brundin P. Parkinson disease epidemiology, pathology, genetics, and pathophysiology. Clin Geriatr Med 2020;36:1-12. 
56 Zesiewicz TA. Parkinson Disease. Continuum (Minneap Minn) 2019;25:896-918.

57. Yokoe M, Mano T, Maruo T, Hosomi K, Shimokawa $\mathrm{T}$, Kishima $\mathrm{H}$, et al. The optimal stimulation site for high-frequency repetitive transcranial magnetic stimulation in Parkinson's disease: A double-blind crossover pilot study. J Clin Neurosci 2018;47:72-8.

58. Brys M, Fox MD, Agarwal S, Biagioni M, Dacpano $G$, Kumar P, et al. Multifocal repetitive TMS for motor and mood symptoms of Parkinson disease: A randomized trial. Neurology 2016;87:1907-15.

59. Cohen OS, Rigbi A, Yahalom G, Warman-Alaluf N, Nitsan Z, Zangen A, et al. Repetitive deep TMS for Parkinson disease: A 3-month doubleblind, randomized sham-controlled study. J Clin Neurophysiol 2018;35:159-65.

60. Trung J, Hanganu A, Jobert S, Degroot C, MejiaConstain B, Kibreab M, et al. Transcranial magnetic stimulation improves cognition over time in Parkinson's disease. Parkinsonism Relat Disord 2019;66:3-8.

61. Chung CL, Mak MK. Effect of repetitive transcranial magnetic stimulation on physical function and motor signs in parkinson's disease: A systematic review and meta-analysis. Brain Stimul 2016;9:475-87.

62. Yang C, Guo Z, Peng H, Xing G, Chen H, McClure $\mathrm{MA}$, et al. Repetitive transcranial magnetic stimulation therapy for motor recovery in Parkinson's disease: A Meta-analysis. Brain Behav 2018;8:e01132.

63. Matsumoto H, Ugawa Y. Repetitive transcranial magnetic stimulation for Parkinson's disease: A review. Brain Nerve 2017;69:219-25.

64. Latorre A, Rocchi L, Berardelli A, Bhatia KP, Rothwell JC. The use of transcranial magnetic stimulation as a treatment for movement disorders: A critical review. Mov Disord 2019;34:769-82.

65. Charlson FJ, Ferrari AJ, Santomauro DF, Diminic S, Stockings E, Scott JG, et al. Global epidemiology and burden of schizophrenia: Findings from the global burden of disease study 2016. Schizophr Bull 2018;44:1195-203.

66. Owen MJ, Sawa A, Mortensen PB. Schizophrenia. Lancet 2016;388:86-97.

67. Dougall N, Maayan N, Soares-Weiser K, McDermott LM, McIntosh A. Transcranial magnetic stimulation for schizophrenia. Schizophr Bull 2015;41:1220-2.

68. Marzouk T, Winkelbeiner S, Azizi H, Malhotra AK, Homan P. Transcranial magnetic stimulation for positive symptoms in schizophrenia: A systematic review. Neuropsychobiology 2019:1-13.

69. Mehta UM, Naik SS, Thanki MV, Thirthalli J. Investigational and Therapeutic applications of transcranial magnetic stimulation in schizophrenia. Curr Psychiatry Rep 2019;21:89.

70. Linsambarth S, Jeria A, Avirame K, Todder D, Riquelme R, Stehberg J. Deep transcranial magnetic stimulation for the treatment of negative symptoms in schizophrenia: Beyond an antidepressant effect. $\mathrm{J}$ ECT 2019;35:e46-e54.
71. Wobrock T, Guse B, Cordes J, Wölwer W, Winterer G, Gaebel W, et al. Left prefrontal high-frequency repetitive transcranial magnetic stimulation for the treatment of schizophrenia with predominant negative symptoms: a sham-controlled, randomized multicenter trial. Biol Psychiatry 2015;77:979-88.

72. Kozel FA. Clinical repetitive transcranial magnetic stimulation for posttraumatic stress disorder, generalized anxiety disorder, and bipolar disorder. Psychiatr Clin North Am 2018;41:433-46.

73. Sagliano L, Atripaldi D, De Vita D, D'Olimpio $\mathrm{F}$, Trojano L. Non-invasive brain stimulation in generalized anxiety disorder: A systematic review. Prog Neuropsychopharmacol Biol Psychiatry 2019;93:31-8.

74. Guo Q, Li C, Wang J. Updated review on the clinical use of repetitive transcranial magnetic stimulation in psychiatric disorders. Neurosci Bull 2017;33:747-56.

75. Miller JN, Black DW. Bipolar Disorder and Suicide: a Review. Curr Psychiatry Rep 2020;22:6.

76. Gold AK, Ornelas AC, Cirillo P, Caldieraro MA, Nardi AE, Nierenberg AA, et al. Clinical applications of transcranial magnetic stimulation in bipolar disorder. Brain Behav 2019;9:e01419.

77. Volkow ND, Li TK. Drugs and alcohol: treating and preventing abuse, addiction and their medical consequences. Pharmacol Ther 2005;108:3-17.

78. Reid AG, Lingford-Hughes AR, Cancela LM, Kalivas PW. Substance abuse disorders. Handb Clin Neurol 2012;106:419-31.

79. Westerhausen D, Perkins AJ, Conley J, Khan BA, Farber M. Burden of substance abuse-related admissions to the medical ICU. Chest 2020;157:61-6.

80. Diana M, Raij T, Melis M, Nummenmaa A, Leggio L, Bonci A. Rehabilitating the addicted brain with transcranial magnetic stimulation. Nat Rev Neurosci 2017; 18:685-93.

81. Azevedo CA, Mammis A. Neuromodulation therapies for alcohol addiction: A literature review. Neuromodulation 2018;21:144-8.

82. Bolloni C, Badas P, Corona G, Diana M. Transcranial magnetic stimulation for the treatment of cocaine addiction: evidence to date. Subst Abuse Rehabil 2018;9:11-21.

83. Hamid P, Malik BH, Hussain ML. Noninvasive transcranial magnetic stimulation (TMS) in chronic refractory pain: A systematic review. Cureus 2019;11:e6019.

84. Barker AT, Shields K. Transcranial magnetic stimulation: Basic principles and clinical applications in migraine. Headache 2017;57:517-24.

85. Young NA, Sharma M, Deogaonkar M. Transcranial magnetic stimulation for chronic pain. Neurosurg Clin N Am 2014;25:819-32.

86. Anninos P, Chatzimichael A, Adamopoulos A, Kotini A, Tsagas N. A combined study of MEG and picoTesla TMS on children with autism disorder. J Integr Neurosci 2016;15:497-513. 
87. Barahona-Corrêa JB, Velosa A, Chainho A, Lopes $\mathrm{R}$, Oliveira-Maia AJ. Repetitive transcranial magnetic stimulation for treatment of autism spectrum disorder: A systematic review and meta-analysis. Front Integr Neurosci 2018;12:27.
88. Meeus OM, De Ridder D, Van de Heyning PH. Transcranial magnetic stimulation (TMS) in tinnitus patients. B-ENT 2009;5:89-100.

89. Hallett M. Transcranial magnetic stimulation: a primer. Neuron 2007;55:187-99. 